УДК 621.39

DOI 10.18413/2687-0932-2020-47-2-422-431

\title{
ПРЕДСТАВЛЕНИЕ СИГНАЛОВ ПРИ ОБРАБОТКЕ ИНФОРМАЦИИ В МАЛОБАЗОВОЙ ПОЛЯРИЗАЦИОННОЙ ИЗМЕРИТЕЛЬНОЙ СИСТЕМЕ
}

\section{MODELS REPRESENTATION SIGNAL IN THE PROCESSING OF INFORMATION IN SMALL BASIC POLARIZING MEASURING SYSTEM}

\author{
И.И. Олейник \\ I.I. Oleynik
}

Белгородский государственный национальный исследовательский
университет, ул. Победы, д. 85, г. Белгород, 308015, Россия

Belgorod National Research University, 85 Pobedy St., Belgorod, 308015, Russia

E-mail: oleinik_i@bsu.edu.ru

\begin{abstract}
Аннотация
C развитием радиолокационной техники, в современных условиях, большую актуальность приобретают МIMO системы. Основным принципом функционирования таких систем является возможность сканирования одного и того же объекта несколькими радиолокаторами, или несколькими радиолокационными позициями, отстоящими друг от друга на расстоянии. При этом обработка отраженных от объекта сигналов заключается не только в определении координат объектов, но и в использовании корреляционных связей между отраженными сигналами с целью получения дополнительных признаков для обнаружения и распознавания объектов. Варианты таких систем, имеющих поляризационные различия в излучаемых и принимаемых сигналах, на сегодняшний день еще недостаточно изучены. В статье рассмотрены структура и принципы функционирования малобазовой поляризационной измерительной системы. Получены математические выражения для описания излучаемых и принимаемых отраженных от объектов сигналов двумя разнесенными позициями, работающих на ортогональных поляризациях. Получены выражения, описывающие напряжения на выходе приемных каналов малобазовой поляризационной измерительной системы при полном поляризационном зондировании для формирования поляризационного вектора рассеяния объекта. Проведен анализ полученных выражений и определены параметры сигналов, влияющие на дальнейшую обработку.
\end{abstract}

\begin{abstract}
With the development of radar technology, in modern conditions, MIMO systems become more relevant. The main principle functioning of such systems is the ability to scan the same object with several radars, or several radar positions that are separated from each other at a distance. In this case, the processing of signals reflected from an object consists not only in determining the coordinates of objects , but also in using correlations between the reflected signals, in order to obtain additional features for detecting and recognizing objects. Variants of such systems, which have polarizing differences in the radiated and received signals, have not yet been sufficiently studied. The article considers the structure and principles of functioning of a small basic polarization measuring system. Mathematical expressions are obtained for describing the signals emitted and received reflected from objects by two separated positions operating on orthogonal polarizations. Expressions describing the voltage at the output of the receiving channels of a small basic polarization measuring system, with full polarization sensing, for the formation of a polarizing scattering vector of the object are obtained. The obtained expressions were analyzed and the signal parameters affecting further processing were determined.
\end{abstract}

Ключевые слова: поляризационная измерительная система, поляризационный вектор, малобазовая система, сигнал, амплитуда, приемный канал, фаза, антенна.

Keywords: polarizing measuring system, polarization vector, small basic system, signal, amplitude, receiving channel, phase, antenna. 


\section{Введение}

На сегодняшний день вопросам обработки информации в многопозиционных радиолокационных измерительных системах уделяется большое внимание. В литературе они описываются как МІМО системы [Черняк, 2011]. Однако принципы формирования, приема и обработки сигналов с поляризационными различиями в таких системах изучены недостаточно [Bliss et al., 2003]. Необходимы математические выражения для корректного описания сигналов с поляризационными различиями. Эти различия проявляются как при излучении сигналов, так и при их приеме. Причем особенно необходимо определить фазовые различия, которые будут проявляться при приеме сигналов. Это обусловлено тем, что образуется разность фаз между принимаемыми сигналами на различных позициях. Причем, чем больше отклонение диаграммы направленности антенны от нормали, тем больше образовывается набег фаз [Li et al., 2009].

Под малобазовой радиолокационной системой будем понимать двухпозиционную систему, для которой антенны обоих позиций находятся в пределах средней ширины лепестка диаграммы обратного рассеяния облучаемого объекта. Фронт падающей на антенны волны считается плоским.

В общем случае, возможны различные варианты построения мало базовых систем. Например, с одной передающей и двумя приемными позициями, или с одной передающей и одной приемной позицией. Существует еще ряд вариантов построения малобазовых систем (даже когда позиций более двух) [Черняк, 2011].

Ниже рассмотрен наиболее показательный и реально осуществимый вариант малобазовой поляризационной измерительной системы (МПИС).

\section{Структура и принцип функционирования малобазовой поляризационной измерительной системы}

МПИС состоит из двух аналогичных позиций. Одна позиция состоит из приемопередающего устройства и антенны. При этом антенна одной позиции может излучать и принимать сигналы одной линейной поляризации. Вторая позиция так же может излучать и принимать сигналы одной линейной поляризации, но ортогональные по поляризации первой позиции [Костин и др., 2011]. Например, это могут быть сигналы вертикальной и горизонтальной поляризации.

В качестве антенны может выступать активная антенная решетка (АФАР) с определенным сектором электронного сканирования диаграммой направленности (ДН) [Ширман, 1998]. При этом алгоритм сканирования должен обеспечивать излучение и прием сигналов с каждой позиции в одном и том же угловом направлении. Это позволяет одновременно принимать отраженный от одного и того же объекта сигнал на обе позиции.

Результатом функционирования такой малобазовой радиолокационной системы должно быть измерение поляризационной матрицы рассеяния (ПМР) или поляризационного вектора рассеяния (ПВР) объекта [Burdanova et al., 2019; Бурданова и др., 2008; Бурданова и др., 2008].

Для обеспечения измерения ПВР необходимо, чтобы были поочередно излучены сигналы с двух позиций. Прием отраженных сигналов необходимо проводить одновременно на обе позиции, для каждого излученного сигнала. В этом случае возможно сформировать ПВР облучаемого объекта [Burdanova et al., 2019; Бурданова и др., 2007]

$$
\overrightarrow{\dot{U}}(t, R)=\left(\dot{U}_{\Gamma \mathrm{B}}(t, R) \quad \dot{U}_{\Gamma \Gamma}(t, R) \quad \dot{U}_{\mathrm{B \Gamma}}(t, R) \quad \dot{U}_{\mathrm{Bв}}(t, R)\right),
$$

где: $U$ - комплексные амплитуды на выходе приемных каналов, индекс г обозначает горизонтальную поляризацию, индекс в - вертикальную, первый индекс при $U$ обозначает 
излучаемую поляризацию, второй - принимаемую (например, $\dot{U}_{\text {вг }}(t, R)$ - был излучен сигнал на вертикальной поляризации, а принят на горизонтальной).

На рис. 1 приведена структура и принцип функционирования, описанной выше малобазовой системы.

Символами П1 и П2 обозначены первая и вторая позиция, соответственно. Символом $O$ обозначен условный фазовый центр. $R$ - расстояние от условного фазового центра до сканируемого объекта. Символами $r_{1}$ и $r_{2}$ обозначены расстояния от антенн до объекта для первой и второй позиции, соответственно. В общем случае направление на объект может не совпадать с перпендикуляром к условному фазовому центру и может составлять некий угол $\alpha$. Расстояние $\Delta r_{1}$ будет равно разности расстояния $R$ и $r_{1}$, а $\Delta r_{2}$ соответствовать разности $R$ и $r_{2}$. Эти расстояния будут определять разности набега фаз отраженного сигнала относительно условного фазового центра, при приеме их на позиции 1 и 2. В общем случае, антенны обоих позиций могут иметь различные характеристики направленности (нормированные диаграммы направленности) $F_{1}(Q)$ и $F_{2}(Q)$ [Ширман, 2007]. Соответственно и коэффициенты усиления (КУ) антенн $G_{1}$ и $G_{2}$ будут отличаться [Бакулев, 2004]. База между позициями характеризуется расстоянием $B$. Таким образом, для малобазовой системы должно выполняться равенство $B \ll R$.

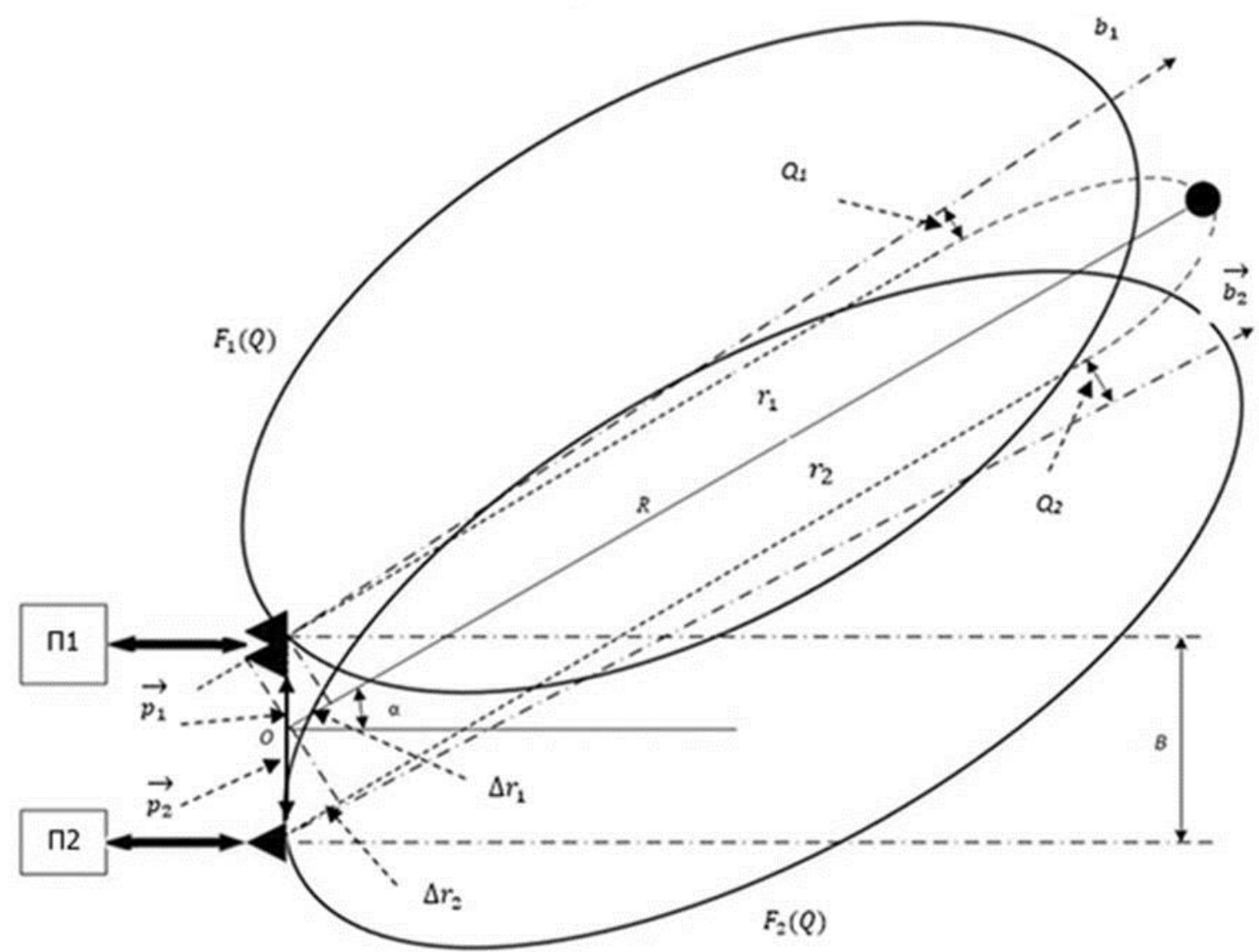

Рис. 1. Структура и принцип функционирования малобазовой поляризационной измерительной системы

Fig. 1. Structure and principle of operation of a small basic polarizing measuring system

\section{Представления сигналов в малобазовой поляризационной измерительной системе}

Рассмотрим принцип и математические описания сигналов при излучении и приеме.

Излученный сигнал $\mathrm{c}$ вертикальной поляризацией может быть записан как напряженность электромагнитного поля в виде [Канарейкин и др., 1966] 


$$
\dot{\overrightarrow{E_{\mathrm{B}}}}(t, R)=\sqrt{60 P_{\mathrm{A}_{\text {пер }}} G_{A_{\text {пер в }}}(\vec{R})} \frac{1}{r_{2}} e^{-j k r_{2}} e^{j \Delta \varphi_{\text {пер в }}} \dot{T}_{\text {пер в }}(t) \overrightarrow{e_{\mathrm{B}}},
$$

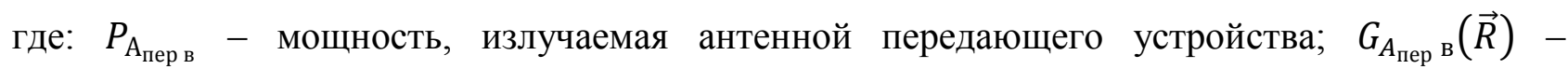
максимальный коэффициент усиления (КУ) антенны на передачу в направлении на объект;

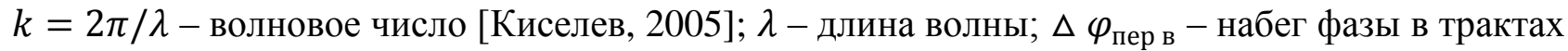
передающего устройства; $\dot{T}_{\text {пер в }}(t)$ - вид сигнала (модуляция комплексной огибающей) [Канарейкин и др., 1968]; $\overrightarrow{e_{\mathrm{B}}}-$ орт поляризации передающей антенны; $r_{2}-$ расстояние от антенны передающего устройства до объекта.

Отраженный от объекта сигнал имеет вид

$$
\overrightarrow{E_{\mathrm{B} 0}}(t, R)=\sqrt{60 P_{\mathrm{A}_{\text {пер в }}} G_{A_{\text {пер в }}}(\vec{R})} \frac{1}{r_{2}} e^{-j k r_{2}} e^{j \Delta \varphi_{\text {пер в }}} \dot{T}_{\text {пер в }}(t)\left(\begin{array}{c}
\sigma_{\mathrm{Bв}} \\
\sigma_{\mathrm{B \Gamma}}
\end{array}\right),
$$

где: $\sigma_{\text {вв }}$ - эффективная поверхность рассеяния объекта на вертикальной поляризации, при облучении его сигналом вертикальной поляризации; $\sigma_{\text {вг }}-$ эффективная поверхность рассеяния объекта на горизонтальной поляризации при облучении его сигналом вертикальной поляризации [Киселев, 2005; Поздняк и др., 1974].

Напряжение на выходе приемного канала вертикальной поляризации (при излученной вертикальной поляризации) может быть представлено в виде [Киселев, 2005]

$$
\begin{aligned}
& \dot{U}_{\text {вв }}(t, R) \approx K_{\text {пр в }} \sqrt{G_{\mathrm{A}_{\text {пр в }}}(\vec{R}) G_{A_{\text {пер в }}}(\vec{R}) P_{\mathrm{A}_{\text {пер в }}}} \frac{1}{r_{2} r_{2}} e^{-j k\left(r_{2}+r_{2}\right)} e^{j \Delta \varphi_{\text {пер в }}} e^{j \Delta \varphi_{\text {пр в }}} \times \\
& \times \dot{T}_{\text {пер в }}(t) \sigma_{\text {вв }}+\dot{U}_{\text {шв }}(t) \text {, }
\end{aligned}
$$

где: $r_{2}$ - расстояние от объекта до антенны приемного устройства; $\dot{U}_{\text {шв }}(t)-$ напряжение собственных шумов приемного канала вертикальной поляризации; $\Delta \varphi_{\text {пр в }}-$ набег фазы в трактах приемного канала вертикальной поляризации; $K_{\text {пр в }}-$ КУ приемного канала вертикальной поляризации; $G_{\mathrm{A}_{\text {пр в }}}(R)-$ КУ приемной антенны вертикальной поляризации в направлении $R$.

Аналогично можем записать напряжение на выходе приемного канала горизонтальной поляризации (при излученной вертикальной)

$$
\begin{aligned}
& \dot{U}_{\text {вг }}(t, R) \approx K_{\text {пр г }} \sqrt{G_{\mathrm{A}_{\text {пр г }}}(\vec{R}) G_{A_{\text {пер в }}}(\vec{R}) P_{\mathrm{A}_{\text {пер в }}}} \frac{1}{r_{1} r_{2}} e^{-j k\left(r_{1}+r_{2}\right)} e^{j \Delta \varphi_{\text {пер в }}} e^{j \Delta \varphi_{\text {пр г }}} \times \\
& \times \dot{T}_{\text {пер в }}(t) \sigma_{\text {вг }}+\dot{U}_{\text {шг }}(t) \text {, }
\end{aligned}
$$

где: $\dot{U}_{\text {шг }}(t)$ - напряжение собственных шумов приемного канала горизонтальной поляризации; $K_{\text {пр г }}-$ КУ приемного канала горизонтальной поляризации;

$G_{\mathrm{A}_{\text {пр г }}}(R)-\mathrm{KУ}$ приемной антенны горизонтальной поляризации в направлении $R ; \Delta \varphi_{\text {пр г }}-$ набег фазы в трактах приемного канала горизонтальной поляризации.

Рассмотрим представления сигналов с другой поляризацией, при излучении другим пунктом (сигнала горизонтальной поляризации). Излученный сигнал горизонтальной поляризации можно записать в виде

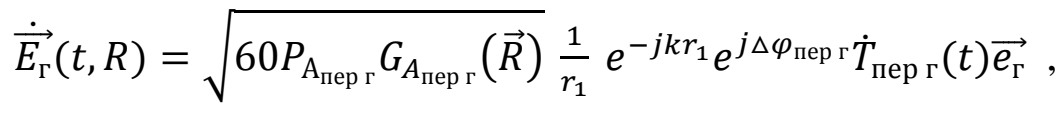

где: $P_{A_{\text {пер г }}}-$ мощность, излучаемая антенной передающего устройства; $G_{A_{\text {перг }}}(\vec{R})-$ максимальный коэффициент усиления (КУ) антенны на передачу в направлении на объект; $k=2 \pi / \lambda-$ волновое число; $\lambda$ - длина волны; $\Delta \varphi_{\text {пер г }}-$ набег фазы в трактах передающего 
устройства; $\dot{T}_{\text {пер г }}(t)$ - вид сигнала (модуляция комплексной огибающей); $\overrightarrow{e_{\Gamma}}-$ орт поляризации передающей антенны; $r_{1}$ - расстояние от антенны передающего устройства до объекта.

Отраженный от объекта сигнал можно записать в виде [Канарейкин и др., 1966]

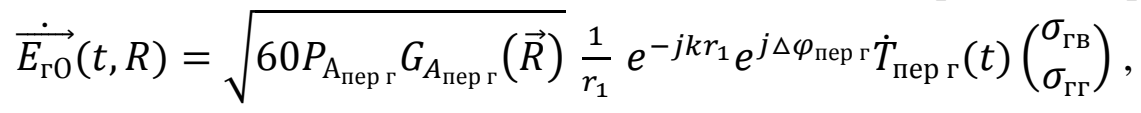

где: $\sigma_{\text {гв }}$ - эффективная поверхность рассеяния объекта на вертикальной поляризации, при облучении его сигналом горизонтальной поляризации [Киселев, 2005];

$\sigma_{\text {гг }}$ - эффективная поверхность рассеяния объекта на горизонтальной поляризации, при облучении его сигналом горизонтальной поляризации;

Напряжение на выходе приемных каналов вертикальной поляризации (при излученной горизонтальной) [Киселев, 2005]

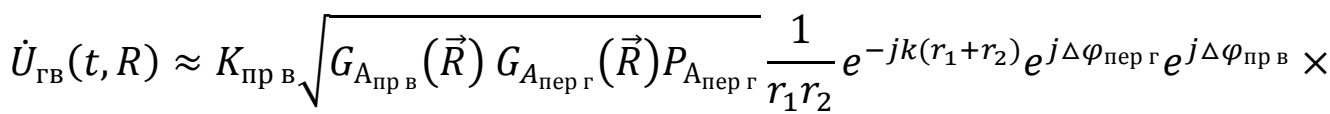

$$
\begin{aligned}
& \times \dot{T}_{\text {пер г }}(t) \sigma_{\text {гв }}+\dot{U}_{\text {шв }}(t),
\end{aligned}
$$

где: $r_{2}$ - расстояние от объекта до антенны приемного устройства; $\dot{U}_{\text {шв }}(t)-$ напряжение собственных шумов приемного канала вертикальной поляризации; $\Delta \varphi_{\text {пр в }}-$ набег фазы в трактах приемного канала вертикальной поляризации; $K_{\text {пр в }}-$ КУ приемного канала вертикальной поляризации; $G_{\mathrm{A}_{\text {пр в }}}(R)-\mathrm{KУ}$ приемной антенны вертикальной поляризации в направлении $R$.

Напряжение на выходе приемных каналов горизонтальной поляризации (при излученной горизонтальной) [Киселев, 2005; Козлов, 1979]

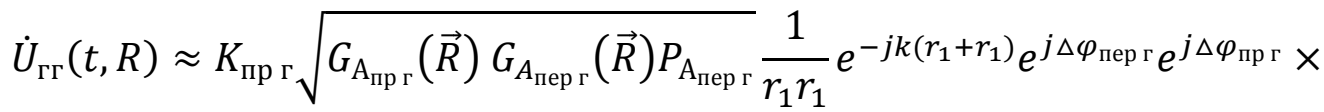

$$
\begin{aligned}
& \times \dot{T}_{\text {пер г }}(t) \sigma_{\text {гг }}+\dot{U}_{\text {шг }}(t),
\end{aligned}
$$

где: $\dot{U}_{\text {шг }}(t)$ - напряжение собственных шумов приемного канала горизонтальной поляризации [Олейник и др., 2006]; $K_{\text {пр г }}-$ КУ приемного канала горизонтальной поляризации; $G_{\mathrm{A}_{\text {пр г }}}(R)-$ КУ приемной антенны горизонтальной поляризации в направлении объекта; $\Delta \varphi_{\text {пр г }}-$ набег фазы в трактах приемного канала горизонтальной поляризации.

Далее введем ряд упрощений и ограничений, обусловленных особенностями МПИС.

При учете амплитуд принимаемых сигналов можно внести допущение, что для расстояния выполняются условия $R \approx r_{1} \approx r_{2}$. Это допущение оправдано тем, что для МПИС эти расстояния составляют километры, а база $B$ - единицы метров. Следовательно, на амплитуду принимаемой волны разница в этих расстояниях не оказывает практически никакого влияния. Тогда для амплитудного множителя имеет место

$$
\frac{1}{R^{2}} \approx \frac{1}{r_{1}^{2}} \approx \frac{1}{r_{2}^{2}} \approx \frac{1}{r_{1} r_{2}}
$$

Для фазовых множителей картина принципиально иная. Общий набег фаз (начало фазы) относительно условного фазового центра $O$ (рис. 1) можно представить в виде

$$
\Psi=2 \cdot k \cdot R
$$

где: $k=2 \pi / \lambda-$ волновое число; $\lambda-$ длина волны. 
Множители $e^{-j k\left(r_{1}+r_{2}\right)}$ в выражениях (4),(5),(8),(9) преобразуются к виду

$$
\exp (-j 2 k R)=\exp (-j \Psi) \text {. }
$$

Определим соотношения для расстояний (рис. 1).

$$
r_{1}=R-\Delta r_{1}, \Delta r_{1}=\rho_{1} \sin \alpha, r_{2}=R+\Delta r_{2}, \Delta r_{2}=\rho_{2} \sin \alpha
$$

Следовательно

$$
-k 2 r_{1}=-k 2 R+k 2 \rho_{1} \sin \alpha, \quad-k\left(r_{1}+r_{2}\right)=-k 2 R-k \sin \alpha\left(\rho_{2}-\rho_{1}\right),
$$

где: $\vec{\rho}_{1}, \vec{\rho}_{2}$ - радиус-векторы положения антенн относительно условного фазового центра $O$.

Таким образом, набеги фаз для отраженных сигналов для антенн обеих позиций можно представить в виде

$$
\begin{gathered}
\Psi_{\text {гГ }}=-k \cdot 2 \rho_{1} \sin \alpha, \\
\Psi_{\mathrm{гB}}=-k \cdot \sin \alpha \cdot\left(\rho_{2}-\rho_{1}\right), \\
\Psi_{\mathrm{B \Gamma}}=k \cdot \sin \alpha \cdot\left(\rho_{2}-\rho_{1}\right), \\
\Psi_{\mathrm{BB}}=k \cdot 2 \rho_{2} \sin \alpha .
\end{gathered}
$$

В выражения (2-9) входят множители $\Delta \varphi_{\text {перг }}, \Delta \varphi_{\text {перв }}, \Delta \varphi_{\text {прг }}, \Delta \varphi_{\text {прв }}$, определяющие величину набега фаз в приемных и передающих каналах при функционировании системы. При обеспечении достаточно высокой синхронизации между пунктами (например, использование общего задающего генератора), эти величины могут быть учтены (или устранены) при настройке аппаратуры [Олейник и др., 2005; Олейник и др., 2007].

Исходя из выше сказанного можно ввести обозначения

$$
\begin{aligned}
& \mathrm{Z}_{\text {гг }}=K_{\text {прг }} \frac{1}{r_{1}^{2}} \sqrt{G_{A_{\text {ПГ Г Г }}}(\vec{R}) \cdot G_{A_{\text {ПЕРГ }}}(\vec{R}) \cdot P_{A_{\text {ПЕРГ }}}}, \\
& \mathrm{Z}_{\text {гв }}=K_{\text {пр в }} \frac{1}{r_{1} r_{2}} \sqrt{G_{A_{\text {ПР в }}}(\vec{R}) \cdot G_{A_{\text {ПЕР Г }}}(\vec{R}) \cdot P_{A_{\text {ПЕР Г }}}}, \\
& \mathrm{Z}_{\text {вг }}=K_{\text {прг }} \frac{1}{r_{1} r_{2}} \sqrt{G_{A_{\text {ПГР Г }}}(\vec{R}) \cdot G_{A_{\text {ПЕР }}}(\vec{R}) \cdot P_{A_{\text {ПЕР в }}}}, \\
& \mathrm{Z}_{\mathrm{вв}}=K_{\text {пр в }} \frac{1}{r_{2}^{2}} \sqrt{G_{A_{\text {ПР B }}}(\vec{R}) \cdot G_{A_{\text {ПЕР B }}}(\vec{R}) \cdot P_{A_{\text {ПЕР в }}}} .
\end{aligned}
$$

Кроме этого, на обеих позициях используются по одной антенне. Т. е. приемная и передающая антенны совмещены [Козлов, 2011]. Следовательно, можем ввести обозначения для коэффициента усиления антенн

$$
G_{A_{\Gamma}}=G_{A_{\text {ПРГ }}}=G_{A_{\text {ПЕР Г }}}, G_{A_{\mathrm{B}}}=G_{A_{\text {ПР }}}=G_{A_{\text {ПЕР в }}} .
$$

При этом [Ширман, 2007]

$$
G_{A_{\Gamma}}=G_{\max A_{\Gamma}} \cdot\left|F_{1}(Q)\right|^{2}, \quad G_{A_{\mathrm{B}}}=G_{\max A_{\mathrm{B}}} \cdot\left|F_{2}(Q)\right|^{2},
$$


где: $F_{1}(Q)$ и $F_{2}(Q)$ в общем случае комплексные нормированные диаграммы направленности (ДН) антенн первой и второй позиции соответственно.

Выражения (19-22) можно привести к виду

$$
\begin{aligned}
& \mathrm{Z}_{\text {гг }}=K_{\text {прг }} \frac{1}{R^{2}} \sqrt{G_{A_{\mathrm{\Gamma}}}^{2}(\vec{R}) \cdot P_{A_{\text {ПЕР Г }}}}, \quad Z_{\text {гв }}=K_{\text {прв }} \frac{1}{R^{2}} \sqrt{G_{A_{\mathrm{B}}}(\vec{R}) \cdot G_{A_{\mathrm{\Gamma}}}(\vec{R}) \cdot P_{A_{\text {ПЕе Г Г }}}}, \\
& \mathrm{Z}_{\mathrm{вв}}=K_{\text {пр в }} \frac{1}{R^{2}} \sqrt{G_{A_{\mathrm{B}}}^{2}(\vec{R}) \cdot P_{A_{\text {ПЕР В }}}}, Z_{\text {вг }}=K_{\text {прг }} \frac{1}{R^{2}} \sqrt{G_{A_{\Gamma}}(\vec{R}) \cdot G_{A_{\Gamma}}(\vec{R}) \cdot P_{A_{\text {ПЕР в }}}} \text {. }
\end{aligned}
$$

С учетом всех допущений и введенных обозначений напряжения на выходе приемных каналов $(4,5,8,9)$ можно записать в виде

$$
\begin{aligned}
& \dot{U}_{\text {гг }}(t, R)=Z_{\text {гг }} \exp \left(-j \Psi_{\text {гг }}\right) \dot{T}_{\text {перг }}(t) \sigma_{\text {гг }}+\dot{U}_{\text {шг }}(t), \\
& \dot{U}_{\text {гв }}(t, R)=Z_{\text {гв }} \exp \left(-j \Psi_{\text {гв }}\right) \dot{T}_{\text {перг }}(t) \sigma_{\text {гв }}+\dot{U}_{\text {шाв }}(t), \\
& \dot{U}_{\mathrm{вг}}(t, R)=Z_{\text {вг }} \exp \left(-j \Psi_{\text {вг }}\right) \dot{T}_{\text {перв }}(t) \sigma_{\text {вг }}+\dot{U}_{\text {шг }}(t), \\
& \dot{U}_{\text {вв }}(t, R)=Z_{\text {вв }} \exp \left(-j \Psi_{\text {вв }}\right) \dot{T}_{\text {перв }}(t) \sigma_{\text {вв }}+\dot{U}_{\text {шв }}(t) .
\end{aligned}
$$

Для корректного излучения и приема сигнала множители, описывающие вид сигнала (модуляция комплексной огибающей), должны удовлетворять условиям

$$
\dot{T}_{\text {перв }}(t)=\dot{T}_{\text {перг }}(t)=\dot{T}(t), \quad \dot{T}(t) \cdot \dot{T}^{*}(t-\tau)=\left\{\begin{array}{cc}
1 & n p u \tau=0 \\
<<1 & \text { nрu } \tau \neq 0
\end{array}\right.
$$

T. е. выражения (27-30) корректны при $\tau=0$.

\section{Анализ свойств сигналов в МПИС}

Полученные выражения позволяют выявить некоторые характерные особенности сигналов при излучении и приеме.

Амплитуды отраженных сигналов (в данном случае имеется в виду энергетика) практически не зависят от структуры малобазовой системы. Это определяется тем, что при $B \ll R$ разницы расстояний (рис. 1) $\Delta r_{1}$ и $\Delta r_{2}$ несоизмеримо малы по сравнению с $R$ и соответственно никакого существенного влияния на амплитуду сигналов влияния не оказывают.

Энергетические характеристики принимаемых сигналов будут, в основном, определяться ЭПР объекта на различных поляризациях (величины $\sigma_{\text {вв }}, \sigma_{\text {вг }}, \sigma_{\text {гв }}, \sigma_{\text {гг }}$ ), то есть непосредственно характеристиками объекта, при отражении от него зондирующих сигналов различных поляризаций.

Кроме этого, на энергетические характеристики сигналов будут оказывать влияние технические характеристики аппаратуры обеих позиций. Это коэффициенты усиления антенн, коэффициенты усиления приемных трактов, мощности излучаемых сигналов, уровень собственных шумов приемных трактов.

С фазовыми характеристиками ситуация обратная. Собственно $\Delta r_{1}$ и $\Delta r_{2}$ как раз и будут определять начальные фазы принимаемых сигналов на разных позициях. Кроме этого, проявляется зависимость начальных фаз от угла отклонения ДН антенн от нормали (угол $\alpha$ на рис. 1). Выражения (15-18) позволяют получить численные выражения для начальных фаз в зависимости от этих переменных и длины волны излучаемых сигналов. Чем меньше длина волны, тем больший сдвиг начальных фаз принимаемых сигналов. 
В общем случае, используя полученную методику расчета фаз, фазовый центр системы можно привязать к любой условной точке в пределах базы $B$, включая центры антенн любой из двух позиций.

\section{Заключение}

Анализ структуры МПИС и полученные представления сигналов с поляризационными различиями позволяют сделать следующие выводы.

Для исключения влияния технических характеристик приемо-передающих трактов в МПИС необходимо создавать такие тракты как можно более идентичными. Если это требование не достижимо, то необходимо априорное знание этих технических характеристик для учета при совместной обработке информации. При этом возможно получение дополнительных признаков для обнаружения и распознавания сигналов, отраженных от объектов, обусловленных характеристиками самих объектов.

Для точного вычисления взаимных корреляций принимаемых сигналов необходима как можно более точная синхронизация между обеими позициями. Это исключит ошибки в определении разности фаз принимаемых сигналов на различные позиции МПИС. Фактически чем меньше длина волны, тем жестче требования к синхронизации.

\section{Список литературы}

1. Бакулев П.А. 2004. Радиолокационные системы: учебник для вузов. М. Радиотехника: 320.

2. Бурданова Е.В., Денисов А.П., Дикуль О.Д., Новоченко Ю.П., Олейник И.И. 2008. Использование статистических моделей для оценок характеристик радиолокационных систем с поляризационной обработкой информации при принятии решения о наличии объектов на фоне подстилающей поверхности. М. Вопросы радиоэлектроники. Серия: Радиолокационная техника, 2: 56-64.

3. Бурданова Е.В., Денисов А.П., Жиляков Е.Г., Олейник И.И., Синани А.И. 2008. Техническая реализация алгоритмов обнаружения целей на фоне помех в локационных системах с поляризационным зондированием. М. Вопросы радиоэлектроники. Серия: Электронная вычислительная техника, 2: 78-85.

4. Бурданова Е.В., Муромцев В.В., Олейник И.И., Храбростин Д.Б. 2007. Особенности разработки и реализации алгоритмов обработки и отображения информации в обзорной РЛС с полным поляризационным зондированием. Санкт-Петербург. Материалы XXIV Симпозиума: Радиолокационное исследование Природных сред, 6: 98-106.

5. Канарейкин Д.Б., Павлов Н.Ф., Потехин В.А. 1966. Поляризация радиолокационных сигналов. М. Сов. Радио: 440.

6. Канарейкин Д.Б., Потехин В.А., Шишкин И.Ф. Морская поляриметрия. 1968. Л. Судостроение: 328.

7. Киселев А.3. 2005. Теория радиолокационного обнаружения на основе использования векторов рассеяния целей. СПБ. Наука: 295.

8. Козлов А.И. 1979. Свойства статистических параметров элементов матрицы рассеивания радиолокационных целей. Известия вузов. Серия Радиоэлектроника, 22 (1): 14-18.

656.

9. Козлов Н.Н., Лучин А.А., Труфанов Е.Ю. Радиоинформационные системы. 2011. М. Знание:

10.Костин И.Г., Жуков С.А., Копейкин В.В., Едемский Д.Е., Олейник И.И. 2011. Формирование вектора измеряемых параметров для оценки поляризационных различий сигналов в георадарных исследованиях. Белгород. Компьютерные науки и технологии: сборник трудов Второй Международной научно-технической конференции, 3-5 октября: 640-645.

11. Олейник И.И., Бурданова Е.В., Дикуль О.Д., Храбростин Б.В. 2006. Методы обработки информации в радиолокационных системах с различными поляриметрическими режимами работы, с целью увеличения их информативности. Санкт-Петербург. Сборник трудов II международной Научнопрактической конференции: Исследование, разработка и применение Высоких технологий в промышленности, 4: 156-159.

12.Олейник И.И., Дикуль О.Д., Мартынчук А.А., Омельченко А.И., Храбростин Б.В., Храбростин Д.Б. 2005. Радиолокационный комплекс для измерения поляризационных векторов 
рассеяния объектов. Жуковский. Сборник докладов XVIII научно-технической конференции ОАО «НИИ Приборостроения им. В.В. Тихомирова: 263-272.

13.Олейник И.И., Бурданова Е.В., Муромцев В.В. 2007. Особенности разработки и реализации алгоритмов обработки и отображения информации в обзорной РЛС с полным поляризационным зондированием. Санкт-Петербург. Материалы XXIV Симпозиума: Радиолокационное исследование Природных сред, Вып. 6: 245-251.

14.Поздняк С.И., Мелитицкий В.А. 1974. Введение в статистическую теорию поляризации радиоволн. М. Сов. Радио: 480.

15.Черняк В.С. 2011. О новых и старых идеях в радиолокации: МІМО РЛС. Успехи современной радиоэлектроники, 2: 5-20.

16.Ширман Я.Д. 1998. Справочник по радиоэлектронным системам. М. МАКВИС: 422.

17.Ширман Я.Д. 2007. Радиоэлектронные системы: Основы построения и теория: справочник. М. Радиотехника: 512.

18.Bliss D.W., Forsythe K.W. 2003. Multiple-input multiple-output (MIMO) radar and imaging: Degrees of freedom and resolution. Records 37th Asilomar Conf. on Signals, Systems and Computers, Pacific Groove, CA, USA, 1: 54-59.

19.Burdanova E.V., Zhilyakov E.G., Mamatov A.V., Nemtsev A.N., Oleynik I.I. 2019. Decisive rule experimental studies to detect objects on the background of the earth surface using polarization differences of radar signals. COMPUSOFT. An International Journal of Advanced Computer Technology, 8 (6): 3166-3170.

20.Li J., Stoica P. 2009. MIMO radar signal processing. New Jersey: A John Wiley \& sons inc.: 312.

\section{References}

1. Bakulev P.A. 2004. Radar system: textbook for universities. M. Radiotechnics: 320 (in Russian).

2. Burdanova E.V., Muromtsev V.V., Oleynik I.I., Hrabrostin D.B. 2007. Features of development and implementation of algorithms for processing and displaying information in the full polarization sensing radar. Saint-Petersburg. Materials of the symposium XXIV: Radar research of natural environments. Release 6: 98-106 (in Russian).

3. Burdanova E.V., Denisov A.P., Dikul O.D., Novochenko Y.P., Oleynik I.I. 2008. Use of statistical models to estimate the characteristics of polarized radar systems when deciding on the presence of objects against underlying surface. M. Radio electronics questions. Series: Radar equipment. release 2: 56-64 (in Russian).

4. Burdanova E.V., Denisov A.P., Zhilyakov E.G., Oleynik I.I., Sinani A.I. 2008. Technical implementation of algorithms for detection of targets against the background of interference in location systems with polarization probing. M. Radio electronics questions. Series: Electronic computer facilities. 2: 78-85 (in Russian).

5. Kanareykin D.B., Pavlov N.F., Potehin V.A. 1966. Polarization of radar signals. M. Soviet radio: 440 (in Russian).

6. Kanareykin D.B., Potehin V.A., Hsihskin I.F. Marine polarimetry. 1968. L. Shipbuilding: 328 (in Russian).

7. Kiselev A.Z. Theory of radar detection based on the use of target scattering vectors. 2005. SPB. The science: 295 (in Russian).

8. Kozlov A.I. 1979. Properties of statistical parameters of elements dispersion matrix of the radar target. Proceedings of the universities. Series: radio Electronics, t. 22(1): 14-18 (in Russian).

9. Kozlov N.N., Luchin A.A., Trufanov E.Y. 2011. Radio information systems. M. Knowledge: 656 (in Russian).

10.Kostin I.G., Zukov S.A., Kopeykin V.V., Edemskiy D.E., Oleynik I.I. 2011. Generation of vector of measured parameters for assessment of polarization differences of signals in georadar researches. Computer science and technology: compilation of works of the Second International Scientific and Technical Conference. 3-5 october. Belgorod: 640-645 (in Russian).

11.Oleynik I.I., Burdanova E.V., Dikul O.D., Hrabrostin B.V. 2006. Methods of information processing in radar systems with different polarimetric modes of operation, in order to increase their informativeness. Saint-Petersburg. Proceedings of the II international Scientific and practical conference: Research, development and application of High technologies in industry. 4: 156-159 (in Russian).

12.Oleynik I.I., Dikul O.D., Martunchuk A.A., Omelchenko A.I., Hrabrostin B.V., Hrabrostin D.B. 2005. Radar system for measuring the polarization of the scattering vectors of objects. city of Zhukovsky. 
Collection of reports XVIII scientific and technical conferences OAO «NII Instrument making» Tihomirov V.V.: 263-272 (in Russian).

13. Oleynik I.I., Burdanova E.V., Muromtsev V.V. 2007. Features of development and implementation of algorithms for processing and displaying information in a survey radar with full polarizing sensing. Saint-Petersburg. Materials XXIV Symposium's: Radar research of Natural environments, 6: 245-251 (in Russian).

14.Pozdnyak S.I., Melitichkiy V.A. 1974. Introduction to the statistical theory of radio wave polarization. M. Soviet radio: 480 (in Russian).

15.Chernyk V.S. 2011. About new and old ideas in radar: MIMO RLS. Achievements of modern radio electronics, 2: 5-20 (in Russian).

16.Shirman Y.D. 1998. Electronic Systems Reference Book. M. МАКВИС: 422 (in Russian).

17.Shirman Y.D. 2007. Radio electronic systems: Fundamentals of construction and theory: guide. M. Radiotechnics: 512 (in Russian).

18.Bliss D.W., Forsythe K.W. 2003. Multiple-input multiple-output (MIMO) radar and imaging: Degrees of freedom and resolution. Records 37th Asilomar Conf. on Signals, Systems and Computers, Pacific Groove, CA, USA, 1: 54-59.

19.Burdanova E.V., Zhilyakov E.G., Mamatov A.V., Nemtsev A.N., Oleynik I.I. 2019. Decisive rule experimental studies to detect objects on the background of the earth surface using polarization differences of radar signals. COMPUSOFT. An International Journal of Advanced Computer Technology 8(6) 3166-3170.

20.Li J., Stoica P. 2009. MIMO radar signal processing. New Jersey: A John Wiley \& sons inc.: 312.

\section{Ссылка для цитирования статьи For citation}

Олейник И.И. 2020. Представление сигналов при обработке информации в малобазовой поляризационной измерительной системе. Экономика. Информатика. 47 (2): 422-431. DOI: 10.18413/2687-0932-2020-47-2-422-431.

Oleynik I.I. 2020. Models representation signal in the processing of information in small basic polarizing measuring system. Economics. Information technologies. 47 (2): 422-431 (in Russian). DOI: 10.18413/2687-0932-2020-47-2-422-431. 